Revista Brasileira de Cartografia

ISSN 1808-0936 | https://doi.org/10.14393/revbrascartogr

Sociedade Brasileira de Cartografia, Geodésia, Fotogrametria e Sensoriamento Remoto

\title{
Reforma Agrária no Brasil: Classificação Baseado em Objeto e Reflectância Acumulada no Monitoramento e Fiscalização da Terra
}

\section{Agrarian Reform in Brazil: Object-oriented classification and accumulated reflectance in monitoring and enforcement of land}

Ramon Chaves de Araújo ${ }^{1}$, Tati de Almeida ${ }^{2}$, Rejane Ennes Cicerelli ${ }^{3}$ e Suzan Pequeno Rodrigues ${ }^{4}$

\author{
1 Instituto Nacional de Colonização e Reforma Agrária - INCRA, Coordenação-Geral de Cartografia. E-mail: \\ ramon.araujo@incra.gov.br \\ ORCID: https://orcid.org/0000-0002-8747-5839 \\ 2 Universidade de Brasília -UnB, Instituto de Geociências, Brasília, Brasil. E-mail: tati_almeida@unb.br \\ ORCID: https://orcid.org/0000-0002-6387-8254 \\ 3 Universidade de Brasília -UnB, Instituto de Geociências, Brasília, Brasil. E-mail: rejaneig @unb.br \\ ORCID: https://orcid.org/0000-0001-7592-6527 \\ 4 Universidade de Brasília -UnB, Instituto de Geociências, Brasília, Brasil. E-mail: suzanpequeno@ unb.br \\ ORCID: https://orcid.org/0000-0002-3256-8544
}

Recebido: 02.2019| Aceito: 11.2019

Resumo: A automatização do monitoramento e fiscalização do uso e cobertura do solo em projetos de assentamento do Instituto Nacional de Colonização e Reforma Agrária (INCRA) utilizando sensoriamento remoto e técnicas de geoprocessamento podem otimizar, reduzir custos e qualificar as atividades de produtores rurais. Assim, esse trabalho apresenta uma proposta de uso de classificação orientada ao objeto em imagens do satélite Sentinel-2A 1C, a partir da reflectância acumulada e mineração de dados em projetos de assentamento no Bioma Cerrado. Foram empregados softwares livres e gratuitos: QGIS na classificação das imagens Sentinel-2A; InterImage na segmentação das imagens, definição e exportação dos atributos de cada segmento que basearam a classificação e Weka na mineração de dados dos atributos. As classificações com técnica da reflectância acumulada tiveram melhores índices de acurácia (exatidão global e índice Kappa), mas a melhora se deu nas classes relativas à vegetação natural do Bioma Cerrado, não ocorrendo nas classes de Culturas Diversas e Silvicultura. Os programas utilizados executaram de forma satisfatória todos os procedimentos propostos para a consecução dos objetivos do trabalho, demonstrando sua aplicabilidade no monitoramento do projeto de assentamento.

Palavras-chave: Sentinel-2. Classificação Orientada ao Objeto. Reflectância Acumulada. Mineração de Dados.

\begin{abstract}
The use of remote sensing and geoprocessing techniques can optimize time, reduce costs and qualify the monitoring activities of land use and occupation by the National Institute of Colonization and Agrarian Reform (INCRA) in rural settlements. This work was intended to apply and discuss some of these techniques: the objectoriented classification of images of the Sentinel-2 satellite, using accumulated reflectance and data mining. The study was conducted in the areas of Colonia I and Colônia II rural settlements, in the municipality of Padre Bernardo / GO. In this study only free software were used: QGIS in the pre-processing of Sentinel-2 images, vector editions and preparation of the final layout of the classification map; InterImage in the segmentation of the images, definition and export of the attributes of each segment that based the classification and; Weka for the data mining of the chosen attributes, identifying the thresholds of the different classes to be mapped. The classifications with the accumulated reflectance technique improve the accuracy (global accuracy and Kappa index) as a whole, but only occurred in the classes related natural vegetation unlike the classes Cultures and Forestry. The programs used performed satisfactorily all the procedures proposed to achieve the objectives of the work demonstrating their applicability in the monitoring of the settlement projects.
\end{abstract}

Keywords: Sentinel-2. Object-oriented Classification. Accumulated Reflectance. Data Mining. 


\section{INTRODUÇÃO}

A reforma agrária no Brasil, sob responsabilidade do Instituto Nacional de Colonização e Reforma Agrária (INCRA) visa conceder direitos de terra aos pequenos proprietários sob duas variantes: distribuição de terras públicas disponíveis e redistribuição de grandes propriedades (PACHECO, 2009; OLIVEIRA, 2013). Entre 1992 e 2003 cerca de 108,5 milhões de hectares passaram a fazer parte dessa estrutura fundiária, principalmente nos biomas Amazônia e Cerrado (GIARD; FERNANDES, 2008).

O impacto da reforma agrária na distribuição de terras no Brasil ainda é pouco compreendido. Para manter o uso benéfico, os pequenos proprietários devem respeitar as regras vigentes para reduzir impactos sobre os recursos naturais e florestais e proteger as populações indígenas e locais (ALSTON, LIBECAP e MUELLER, 2000; GOULD, 2006; PACHECO, 2009). Assim, para examinar a eficiência das práticas e políticas do governo e melhorar os programas de regularização, é necessário desenvolver sistemas de monitoramento e controle visando acompanhar a efetividade de políticas públicas adotadas para a população assentada, tais como crédito agrícola e assistência técnica (INCRA, 2017; GOULD, 2006; McLAUGHIN; McKENNA, 1998).

O sensoriamento remoto é uma ferramenta eficaz para estudos de controle do uso e cobertura da terra (SCHOWENGERDT, 2012), especialmente do Brasil que possui uma extensão continental, seis biomas e tipos de ocupação variados. Assim, a extração de informações automáticas, em que o intérprete tem interferência mínima no processo, poderá otimizar a identificação da realidade produtiva e ambiental em parcelas de assentamentos e orientar áreas prioritárias a serem geridas pelo governo. Neste contexto a utilização de metodologias de Análise de Imagens Baseada em Objetos Geográficos (GEOBIA) vem sendo empregada, nas últimas décadas, para classificação do uso e cobertura da terra, sendo mais uma opção para identificação de feições da superfície terrestre, assim como a mineração de dados. Reforça-se que Projetos de Assentamentos (PA), correspondem a um tipo de uso do solo que envolve associações, em mosaico, de diversas modalidades de uso e cobertura da terra que, em função da escala de trabalho e de características físicas das imagens de satélite adotadas, as imagens possuem similaridades na resposta espectral dificultando o processo de discriminação individual das classes temáticas. Nesse caso, aprimorar técnicas e protocolos de processamento de imagens, adotando a utilização de softwares livres são essenciais em termos sociais e científicos (NASCIMENTO; SANO, 2010).

Assim, esse trabalho utilizou dados do sensor Sentinel2A e classificação orientada a objetos geográficos, na qual a distinção entre alvos não se resume somente na característica espectral do pixel, mas também na mineração de dados, em conjunto com a reflectância acumulada, ou seja a soma aritmética das bandas espectrais de imagens de sensores orbitais, podem potencializar a capacidade de discriminação entre os alvos de um assentamento rural.

\section{MATERIAL E MÉTODO}

A área de estudo está localizada no bioma Cerrado, município de Padre Bernardo, dentro da Área de Proteção Ambiental (APA) do Descoberto (Figura 1). Dois projetos de assentamento (Colônia I e Colônia II) foram implementados em meados de 1990 com respectivamente 595,81 e 577,23 hectares e 23 famílias assentadas cada (Figura 1). As parcelas (lotes) de cada família foram delimitadas, existindo áreas coletivas destinadas à reserva legal ambiental e à produção familiar. As atividades produtivas são diversificadas, com destaque para horticultura e fruticultura, em sua maioria, orgânicos. Esses assentamentos são referências de produção no DF.

Utilizando as classes de uso e cobertura da terra (nível II), definidas pelo Instituto Brasileiro de Geografia e Estatística (IBGE, 2013), as imagens do satélite Sentinel 2A utilizadas nesse trabalho foram classificadas em Culturas Diversas, Silvicultura, Pastagens, Cerrado Campestre/Arbóreo e Cerrado Formação Florestal. As imagens foram obtidas no nível de processamento $1 \mathrm{C}$, isto é, imagens convertidas para reflectância no topo da atmosfera (TOA) em cinco datas (13 de maio, 01 de agosto, 11 de agosto, 10 de setembro e 20 de setembro de 2016) visando a identificação das atividades produtivas diversificadas da área e à inexistência de nuvens. Em função da grande escala de trabalho, foram utilizadas as bandas espectrais de 
resolução espacial de 10 m (B2 vermelho/B3 verde/B4 azul/ B8 infravermelho próximo) (ESA, 2016). O procedimento para a geração da reflectância acumulada foi executado pelo semi automatic classification plugin do software QGIS.

Figura 1 - Mapa de localização da área de estudo com subdivisão dos dois projetos de assentamento (Colônia I e Colônia II) nas proximidades da sede municipal de Padre Bernardo. Imagem de fundo: Banda 4 do satélite Sentinel $2 \mathrm{~A}$.

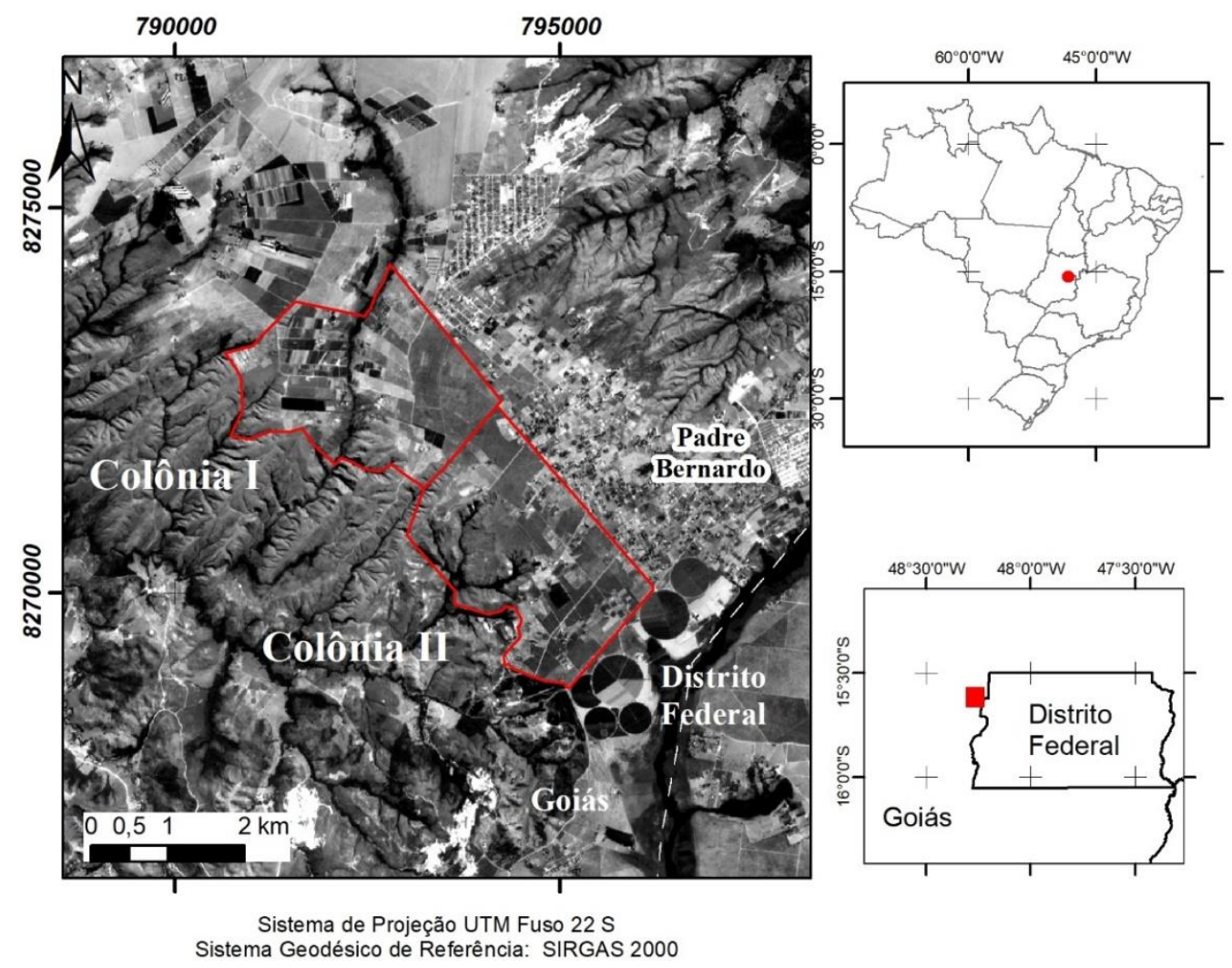

Fonte: Os autores (2020).

Primeiramente, para a análise comparativa da eficiência da reflectância acumulada em áreas de assentamento foram elaborados três conjuntos de imagens: (i) imagem A, cena de 13 de maio de 2016; (ii) Imagem B, reflectância acumulada de duas datas (13 de maio de 2016 e 20 de setembro de 2016); e (iii) Imagem $\mathrm{C}$, reflectância acumulada das cinco datas. Após essa etapa, esses conjuntos de imagens foram segmentados no software InterImage, utilizando-se o segmentador proposto por Baatz e Schäpe (2000). Os parâmetros utilizados na segmentação por regiões estão apresentados na Quadro 1.

Quadro 1 - Parâmetros utilizados na segmentação para a coleta de amostras e na classificação orientada a objeto da imagem do sensor Sentinel 2A de reflectância acumulada.

\begin{tabular}{|l|l|}
\hline \multicolumn{1}{|c|}{ Parâmetros } & \multicolumn{1}{c|}{ Segmentação para coleta de amostras } \\
\hline Operador & TA Baatz Segmenter \\
\hline Bandas & $0,1,2,3$ (referentes às bandas 2, 3, 4 e 8 do Sentinel-2A) \\
\hline Peso das Bandas & $1,1,1,1$ \\
\hline Peso para Compacidade & 1 \\
\hline Peso para Cor & 1 \\
\hline Escala & 1 \\
\hline Otimização & Não \\
\hline Confiança & 0.2 \\
\hline Distância Euclidiana & 20 \\
\hline Operador BottomUp $(1)$ & Dummy BottomUp \\
\hline Ponto de parada & Nenhum \\
\hline
\end{tabular}

Fonte: Os autores (2020). 
Foram escolhidas amostras, considerando uma boa regularidade a partir do método estratificado não alinhado e uma maior representatividade das cinco classes temáticas definidas. Dentre os atributos disponíveis no programa InterImage, extraíram-se os atributos espectrais relevantes para refletir o possível efeito da acumulação da reflectância nas análises. Assim, foram obtidos, por banda e por classe, os seguintes atributos espectrais: MED (valor médio de pixels), PIXMAX (valor máximo de pixel por banda), PIXMIN (valor mínimo de pixel por banda) e MOD (valor mais frequente de pixels por banda - Moda). Em seguida, executouse a mineração dos dados por meio do software Weka 3.6, algoritmo J48 (ANTUNES et al., 2015). Para a seleção da melhor combinação de atributos, os quais supracitados, foram realizados vários testes.

Para a execução da classificação foi inserido no aplicativo QGIS (QGIS, 2016), foi inserido o arquivo vetorial da segmentação e atributos, exportados inicialmente pelo InterImage. Por meio da ferramenta "seleção por expressão" (script) foram selecionados os segmentos de acordo com os limiares apresentados na árvore de decisão fornecida pelo aplicativo Weka. Identificadas as feições, foi incluído um atributo denominado "classe" com a informação nominal de cada classe à qual pertenciam. Ao final deste procedimento, todos os segmentos foram devidamente identificados e classificados em cada classe de interesse. Para alguns procedimentos foi necessária a realização de correções topológicas no arquivo vetorial oriundo do aplicativo InterImage.

A avaliação da acurácia da classificação foi apoiada na matriz de confusão e índices de precisão derivados dela, método padrão para estimar a precisão temática de mapas de uso e cobertura da terra (CONGALTON; GREEN, 1999). Para a geração da matriz de confusão, foram obtidos 150 elementos amostrais (30 pontos por classe), utilizando amostragem sistemática não alinhada. Para a determinação da verdade terrestre nesses pontos foi realizada uma identificação visual utilizando imagens do Google Earth Pro (Digital Globe/CNES Astrium) em conjunto com imagens do satélite Pleiades (resolução espacial de 1,5 metros) de maio de 2016 e do mosaico de levantamento aerofotogramétrico realizado pelo Governo do Distrito Federal em 2015, com resolução espacial de $25 \mathrm{~cm}$. Para testar a significância da diferença entre os índices calculados, aplicou-se o teste Z (CHAGAS et al., 2009; ARRUDA; DEMATTÊ; CHAGAS, 2013). Este teste permite a verificação da significância de apenas um índice ou a diferença entre dois índices.

\section{RESULTADOS E DISCUSSÃO}

Após a realização de diversos testes, optou-se pela combinação dos atributos espectrais MED (valor médio de pixels), PIXMAX (valor máximo de pixel por banda), PIXMIN (valor mínimo de pixel por banda) e MOD (valor mais frequente de pixels por banda). A Tabela 1 apresenta a estatística das árvores de decisão geradas pela mineração de dados, operada sobre cada atributo e sobre as associações de atributos provenientes das amostras de segmentos coletados por classe de uso e cobertura da terra dos grupos de imagens: (i) imagem A, cena de 13 de maio de 2016; (ii) Imagem B, reflectância acumulada utilizando duas datas (13 de maio de 2016 e 20 de setembro de 2016); e (iii) imagem C, reflectância acumulada das cinco datas (13 de maio, 01 de agosto, 11 de agosto, 10 de setembro e 20 de setembro de 2016).

Foram priorizadas imagens representativas das quatro estações do ano, sem nuvens, porém em função da baixa qualidade das imagens do período estudado foi possível adquirir apenas imagens para os meses de maio, agosto e setembro. De fato, a fenologia das culturas influencia na resposta espectral dos alvos, infelizmente a aquisição de satélites são limitadas a algumas épocas do ano (ex.:nuvens).

Tabela 1 - Estatística das árvores de decisão geradas pela mineração de dados com o número de caminhos que culminam nas classes com a combinação dos atributos MED, PIXMAX, PIXMIN e MOD.

\begin{tabular}{l|c|c|c}
\hline \multicolumn{1}{c|}{ Classes } & Imagem A & Imagem B & Imagem C \\
\hline Culturas Diversas & 4 & 2 & 2 \\
Silvicultura & 3 & 3 & 1 \\
Pastagens & 3 & 2 & 2 \\
Cerrado Campestre/Arbóreo & 2 & 2 & 2 \\
Cerrado Formação Florestal & 1 & 1 & 7 \\
Número de atributos & 11 & 7 & \\
\hline
\end{tabular}

Fonte: Os autores (2020)

Analisando a Tabela 2, fica evidente que quanto maior o incremento de imagens (reflectância 
acumulada) a árvore de decisão torna-se mais simples, com menos ramificações. De acordo com Camargo et al. (2012), Novack et al. (2014) e Grande, Almeida e Cicerelli (2016), normalmente as árvores de decisão de menor tamanho e de ramos são consideradas, a priori, mais adequados para subsidiar a classificação. Este aspecto também facilita a operacionalização da classificação, tornando mais ágil a etapa de construção da rede semântica de seleção das classes.

A Figura 2 mostra a configuração da árvore de decisão da associação de atributos da mineração de dados da Imagem C, obtidas a partir da metodologia proposta. Os atributos e regras foram utilizadas nas regras de decisão.

Figura 2 - Árvore de decisão gerada pela mineração de dados operada sobre a associação dos atributos espectrais (MINPIX, MED, MOD, MAXPIX) provenientes das amostras de segmentos coletados por classe de uso e cobertura da terra na imagem $\mathrm{C}$, considerada a priori, a mais adequados para subsidiar a classificação.

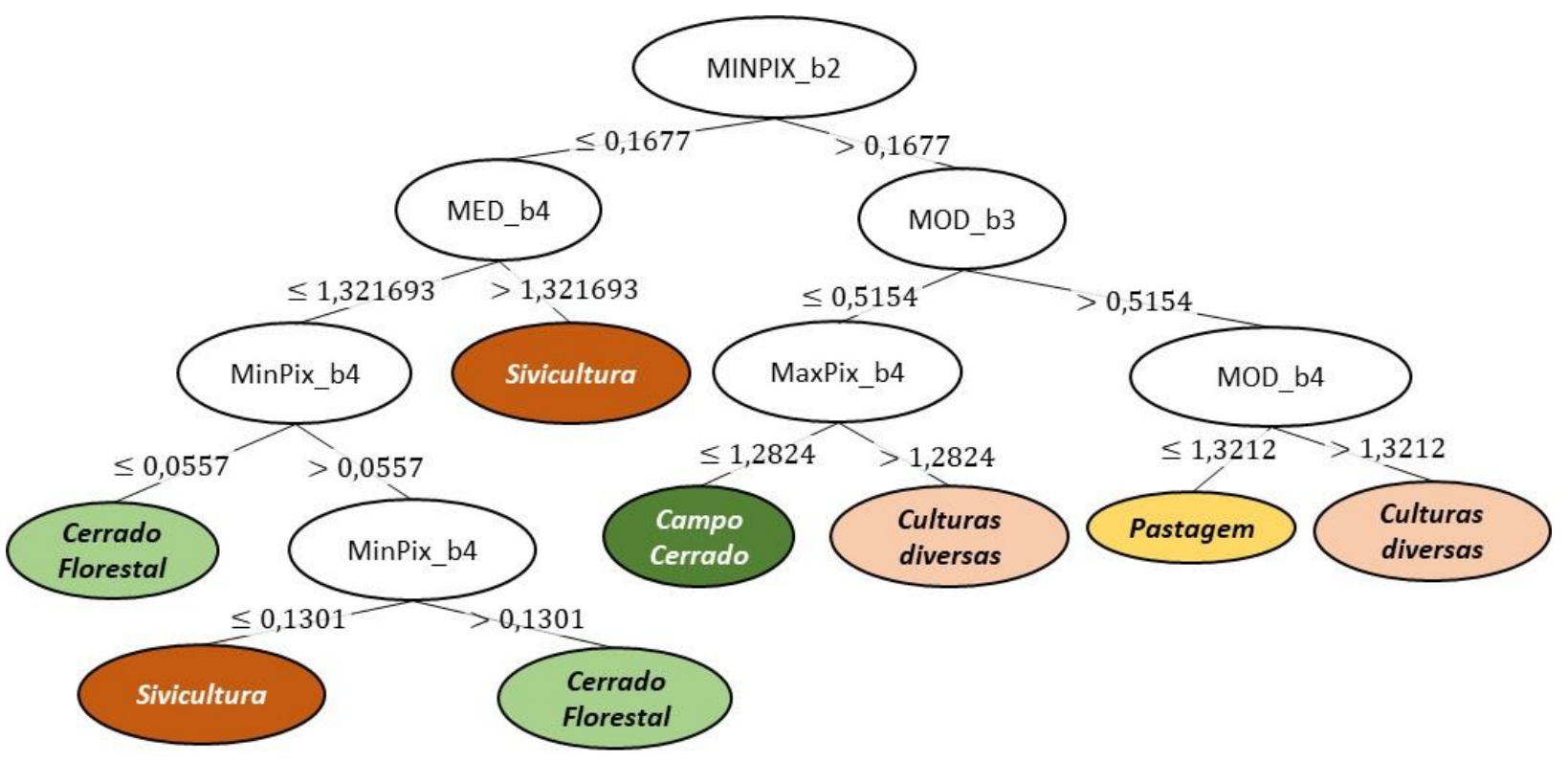

Fonte: Os autores (2020).

Após a seleção das árvores de decisão, a próxima etapa foi gerar as classificações no QGIS por meio da criação de scripts na ferramenta "Seleção por expressão" com base nas regras de decisão estabelecidas na árvore de decisão. O objetivo dessa etapa foi tentar evitar os problemas de limitação na capacidade de processamento encontrados no programa InterImage, citados nos trabalhos de Nascimento et al. (2013), Passo et al. (2013) e Grande, Almeida e Cicerelli (2016). Após uma série de processamentos, o aplicativo foi capaz de gerar as classificações. A Figura 3 apresenta os resultados obtidos após a classificação utilizando as três árvores de decisão.

Os resultados obtidos na distinção dos alvos, com uso de reflectância acumulada de Culturas Diversas, Silvicultura, Pastagens, Cerrado Campestre, Arbóreo e Cerrado Formação Florestal mostraram que a reflectância acumulada foi capaz de discriminar significativamente entre os níveis de acumulação das datas. Resultados similares foram obtidos por Nascimento e Sano (2010), Hermuche e Sano (2011) e Grande, Almeida e Cicerelli (2016), indicando que o manuseio da reflectância acumulada permite maior diferenciação espectral entre os alvos analisados.

No entanto, analisando o processo para cada classe (Tabela 2), identifica-se que a melhora expressiva na classificação se deu de forma mais representativa nas classes de vegetação nativa do cerrado: Cerrado Campo Arbóreo e Cerrado Florestal. A classe Cerrado Campo Arbóreo, por exemplo, apresentou um percentual de acerto crescente, à medida que foram incluídas mais datas no procedimento de reflectância acumulada: $46,15 \%$ na classificação de uma data, $73,08 \%$ na classificação de duas datas e 76,92\% na classificação de cinco datas. O mesmo ocorreu para a classe Cerrado Formação Florestal: 73,68\% de acerto na classificação de uma data, $84,21 \%$ de acerto na classificação de duas datas e $86,84 \%$ na classificação de cinco 
datas. Isso corrobora os resultados encontrados nos trabalhos que avaliaram a reflectância acumulada na discriminação de alvos do Cerrado (e.g., HERMUCHE et al.,2011; GRANDE; ALMEIDA; CICERELLI, 2016). Já a classe "Pastagens", apresentou acerto de $82,81 \%$ de acerto na classificação de uma data (Imagem A), caindo para 73,91\% na classificação da reflectância acumulada em duas datas (Imagem B), e chegando a $100 \%$ na classificação da reflectância acumulada de cinco datas Tais classes de informação sempre foram consideradas complexas para discriminação em função da semelhança espectral desses alvos (GRANDE; ALMEIDA; CICERELLI, 2016; SANO et al., 2010).

Figura 3 - Mapas de uso e ocupação do solo dos projetos de assentamento Colônia I e Colônia II, município de Padre Bernardo/GO, utilizando três árvores de decisão. (a) imagem A com somente a cena de 13 de maio de 2016; (b) Imagem B de reflectância acumulada utilizando duas datas (13 de maio e 20 de setembro de 2016); e (c) Imagem C de reflectância acumulada das cinco datas ( 13 de maio, 01 de agosto, 11 de agosto, 10 de setembro e 20 de setembro de

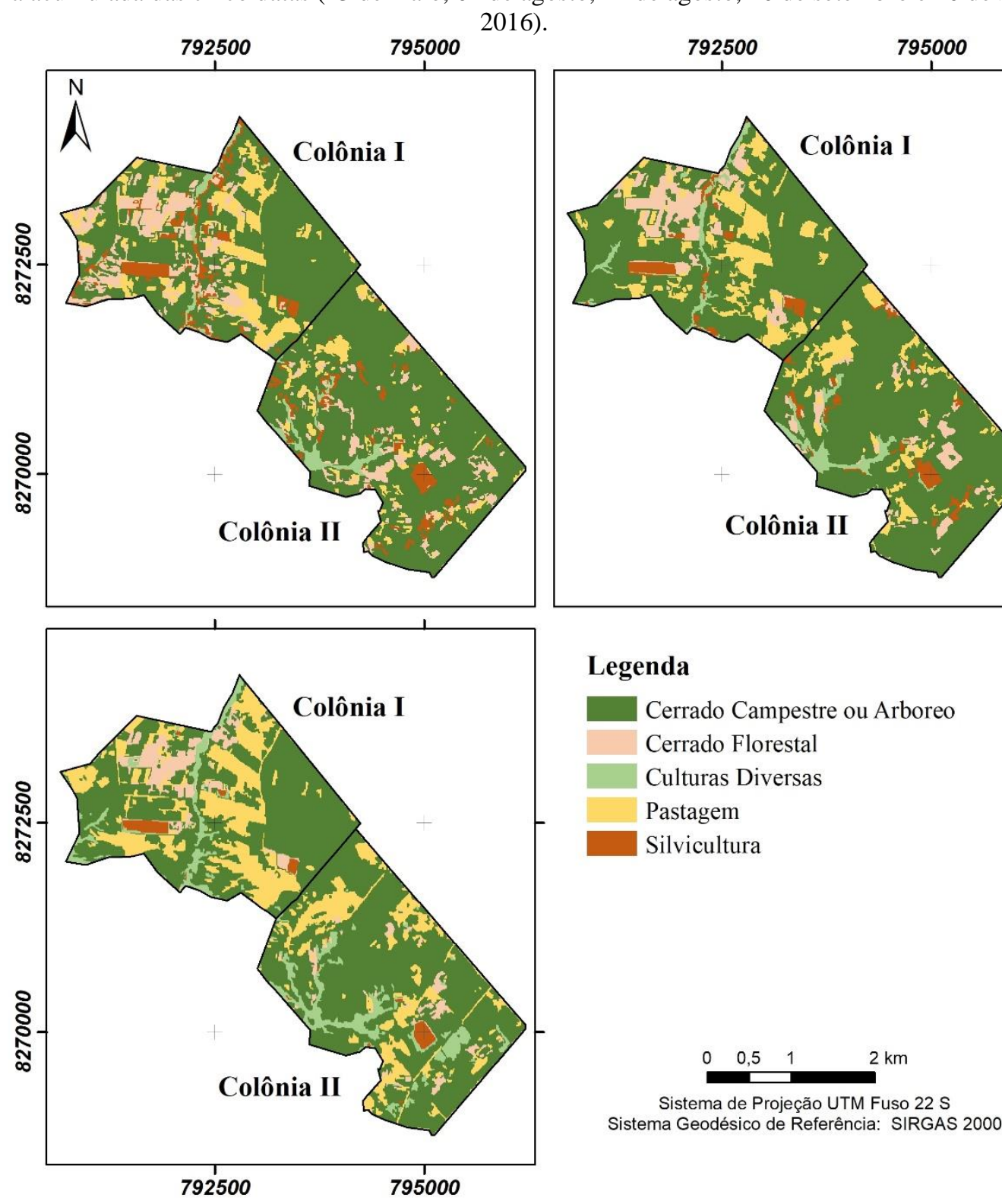

Fonte: Os autores (2020).

Entretanto, para as classes Culturas Diversas e Silvicultura, não houve aumento na acurácia à medida em que se aumenta a reflectância acumulada. A classe Culturas Diversas apresentou uma diminuição na acurácia: 62,96\% de acerto na classificação de uma data (Imagem A), 59,26\% de acerto na classificação da reflectância acumulada de duas datas (Imagem B) e 55,56\% na classificação da reflectância acumulada de cinco datas (Imagem C). Isso ocorreu porque estavam presentes nesta classe tanto culturas perenes quanto culturas temporárias, sendo que a paisagem no local destas últimas sofre grandes variações em curto período 
de tempo, com as imagens captando inclusive o solo completamente exposto onde em meses anteriores estavam plenamente estabelecidas certas culturas. Neste sentido, a avaliação inicial de que não seria necessário realizar a distinção em "Culturas Temporárias" e "Culturas Perenes", se mostrou equivocada. Uma alternativa a ser testada para minimizar esse problema ao se utilizar a técnica de reflectância acumulada é, no momento de selecionar as amostras para a Classe "Culturas Temporárias", escolher intencionalmente áreas onde se faz presente o solo ainda em preparo, conjuntamente com amostras destas culturas em fase inicial e plenamente estabelecidas, para assim representá-la melhor.

Tabela 2 - Matriz de confusão das classificações utilizando três árvores de decisão.

\begin{tabular}{|c|c|c|c|c|c|c|c|c|}
\hline & \multirow[t]{2}{*}{ CLASSIFICAÇÃOO } & \multicolumn{5}{|c|}{ REFERÊNCIA } & \multirow{2}{*}{ Total } & \multirow{2}{*}{$\%$ acerto } \\
\hline & & Culturas & Silvicult & Pastagem & Cer Camp Arb & Cer Florest & & \\
\hline \multirow{8}{*}{ 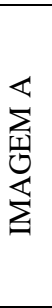 } & Culturas & 17 & 2 & 1 & 10 & 1 & 31 & 62,96 \\
\hline & Silvicultura & 5 & 8 & & 10 & 9 & 32 & 80,00 \\
\hline & Pastagens & 3 & - & 19 & 8 & - & 30 & 82,81 \\
\hline & Cerrado Campo Arbóreo & 2 & - & 3 & 24 & - & 29 & 46,15 \\
\hline & Cerrado Florestal & & - & - & - & 28 & 28 & 73,68 \\
\hline & Total & 27 & 10 & 23 & 52 & 38 & 150 & \\
\hline & \multicolumn{7}{|c|}{ Índice Kappa } & $55 \%$ \\
\hline & \multicolumn{7}{|c|}{ Exatidão Global } & $64 \%$ \\
\hline \multirow{8}{*}{$\sum_{\substack{\mathbb{1} \\
ٍ}}^{\infty}$} & Culturas & 16 & 1 & 1 & 5 & - & 23 & 59,26 \\
\hline & Silvicultura & 1 & 8 & - & 2 & 3 & 14 & 80,00 \\
\hline & Pastagens & 4 & - & 17 & 5 & - & 26 & 73,21 \\
\hline & Cerrado Camp Arb & 5 & 1 & 5 & 38 & 3 & 52 & 73,08 \\
\hline & Cerrado Florestal & 1 & - & - & 2 & 32 & 35 & 84,21 \\
\hline & Total & 27 & 10 & 23 & 52 & 38 & 150 & \\
\hline & \multicolumn{7}{|c|}{ Índice Kappa } & $66 \%$ \\
\hline & & & Exat & o Global & & & & $74 \%$ \\
\hline \multirow{8}{*}{ 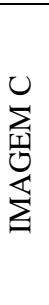 } & Culturas & 15 & 2 & - & 1 & 1 & 19 & 55,56 \\
\hline & Silvicultura & - & 7 & - & - & 3 & 10 & 70,00 \\
\hline & Pastagens & 7 & - & 23 & 11 & - & 41 & 100 \\
\hline & Cerrado Camp Arb & 5 & - & - & 40 & 1 & 46 & 76,92 \\
\hline & Cerrado Florestal & - & 1 & - & - & 33 & 34 & 86,84 \\
\hline & Total & 27 & 10 & 23 & 52 & 38 & 150 & \\
\hline & \multicolumn{7}{|c|}{ Índice Kappa } & $72 \%$ \\
\hline & \multicolumn{7}{|c|}{ Exatidão Global } & $79 \%$ \\
\hline
\end{tabular}

A classe Silvicultura apresentou acerto de $80 \%$ tanto na classificação de uma data (Imagem A), quanto na classificação da reflectância acumulada em duas datas (Imagem B), com este percentual caindo para 70\% na classificação da reflectância acumulada de cinco datas (Imagem C).

Portanto, o aumento da acurácia com o incremento da reflectância acumulada se deu devido à melhoria de acerto nas classes de vegetação nativa (Cerrado Campo/Arbóreo e Cerrado Formação Florestal), por estas comporem a maior parte da área avaliada, e não devido a uma melhoria na discriminação em todos os alvos.

A partir dos índices Kappa e a variância de cada conjunto testado, foi calculada uma matriz de significância de Kappa (Tabela 2). Assim, foi possível comparar os diferentes resultados para a imagem padrão (Imagem A) e as de reflectância acumulada (Imagens B e C). A Tabela 3 mostra, ainda, que todos os valores da diagonal são maiores que $\mathrm{Z}$ tabelado $(1,96)$, indicando que todas as classificações tiveram resultados significativos. O melhor desempenho foi obtido com a utilização da imagem $C$ : cinco datas, que se referem a reflectância acumulada das datas de 13 de maio, 01 de agosto, 11 de agosto, 10 de setembro e 20 de setembro de 2016 ( $\mathrm{Z}$ calculado de 3,63), sendo essa significativamente diferente de todas as demais imagens. 
Tabela 3 - Matriz de significância de Kappa para as classificações realizadas de reflectância (*Existe diferença significativa a nível de 95\%).

\begin{tabular}{c|c|c|c}
\hline Conjunto & Imagem A & Imagem B & Imagem C \\
\hline Exatidão Geral & 64 & 74 & 79 \\
Kappa & 0,55 & 0,66 & 0,72 \\
Variância & 0,049179 & 0,046223 & 0,03764 \\
Imagem A & $2,43^{*}$ & $6,08^{*}$ & $9,38^{*}$ \\
Imagem B & $6,27^{*}$ & $2,99^{*}$ & $3,79^{*}$ \\
Imagem C & $7,07^{*}$ & $2,53^{*}$ & $3,63^{*}$ \\
\hline
\end{tabular}

Fonte: Os autores (2020)

\section{CONCLUSÕES}

A classificação orientada ao objeto, aliada a técnica de mineração de dados se mostraram ser eficientes ferramentas para detecção automatizada do uso e ocupação do solo. A reflectância acumulada tem potencial no aumento da discriminação de determinadas classes, mas sua aplicação exige o conhecimento das alterações da paisagem. Recomenda-se a obtenção de imagens em períodos fenológicas culturais distintas na tentativa de aumentar o potencial de discriminação das classes estudadas.

As classificações executadas nos softwares livres QGIS, InterImage e Weka, apesar de morosas, foram capazes de executar os processamentos sem problemas operacionais. Como proposta para trabalhos futuros, os autores recomendam a criação de um módulo específico para classificação no QGIS com base em árvore de decisão.

A utilização da técnica apresentada, apesar de obter sucesso na classe "nativa", não se mostrou adequada para a fiscalização de culturas sazonais e silvicultura. Tal característica pode ter sido ocasionada pela falta de cenas em períodos sazonais distintos, o que não garantiu o registro espectral de fases diferenciadas de desenvolvimento das culturas. Ainda, fica evidente que o trabalho poderá ser estendido sem grandes restrições, para outras áreas públicas ou privadas, uma vez que o resultado foi satisfatório, mas sempre atentando para a sazonalidade das imagens. Assim, nos projetos de assentamentos rurais propõem-se um estudo metodológico levando em consideração dados com melhores resoluções espacial, temporal e espectral.

\section{Agradecimentos}

Ao curso de Especialização em Geoprocessamento Ambiental da Universidade de Brasília.

\section{Contribuição dos Autores}

O autor Ramon Araújo fez a análise formal, metodologia do projeto a redação - minuta inicial. As autoras Tati Almeida e Rejane Cicerelli supervisionaram, validaram os resultados e redigiram (revisão). A autora Suzan Rodrigues ajudou na análise formal (estatística) e na revisão final do artigo.

\section{Conflitos de Interesse}

Os autores declaram que não há conflitos de interesse.

\section{Referências}

ALSTON, L. J.; LIBECAP, G. D.; MUELLER, B. Land reform policies, the sources of violent conflict, and implications for deforestation in the Brazilian Amazon. Journal of environmental economics and management, v. 39, n. 2, p. 162-188, 2000.

ANTUNES, R.R.; BIAS, E. DE S.; BRITES, R.S.; DA COSTA, G.A.O.P. Desenvolvimento de técnica para monitoramento do cadastro urbano baseado na classificação orientada a objetos. Estudo de caso: município de Goianésia-Go. Revista Brasileira de Cartografia, v. 67, p. 357-372, 2015.

ARRUDA, G. P., DEMATTÊ, J. A. M., CHAGAS, C. S. Mapeamento digital de solos por redes neurais 
artificiais com base na relação solo-paisagem. Revista Brasileira Ciência do Solo, v. 37, p.327-338, 2013.

BAATZ, M.; SCHÄPE, A. Multiresolution segmentation: an optimization approach for high quality multiscale image segmentation. IN: ANGEWANDTE GEOGRAPHISCHE INFORMATIONSVERARBEITUNG, 12., 2000, Salzburg. Salzburg: Wichmann, 2000. p.12-23. Agit Symposium. Edited Josef Strobl, Thomas Blaschke, Gerald Griesebner.

CAMARGO, F.F.; ALMEIDA, C.M.; COSTA, G.A.O.P.; FEITOSA, R.Q.; OLIVEIRA, D.A.B.; HEIPKE, C.; FERREIRA, R.S. An open source object-based framework to extract landform classes. Expert Systems with Applications, v. 39, p. 541-554, 2012. DOI: 10.1016/j.eswa.2011.07.044.

CHAGAS, S. C., VIEIRA, C. A. O., FILHO, E. I. F., JÚNIOR, W. C. Utilização de redes neurais artificiais na classificação de níveis de degradação em pastagens. Revista Brasileira Engenharia Agrícola Ambiental, v. 13, n. 3, p.319-327, 2009.

CONGALTON, R.G.; GREEN, K. Assessing the accuracy of remotely sensed data: principles and practices. Boca Raton: CRC, 1999.137p.

EUROPEAN SPACE AGENCY (ESA). Earth Observation Data. Disponível em: https://sentinel.esa.int/web/sentinel/user-guides/sentinel-2-msi . Acesso em: dezembro de 2016.

GIRARDI, E. P.; FERNANDES, B. M. A luta pela terra e a política de assentamentos rurais no Brasil: a Reforma Agrária conservadora. Agrária (São Paulo. Online), v. 8, p. 73-98, 2008.

GOULD, K. A. Land regularization on agricultural frontiers: The case of Northwestern Petén, Guatemala. Land Use Policy, v. 23, n. 4, p. 395-407, 2006.

GRANDE, T. O.; ALMEIDA, T.; CICERELLI, R. E. Classificação orientada a objeto em associação às ferramentas reflectância acumulada e mineração de dados. Pesquisa Agropecuária Brasileira, Brasília, v. 51, n. 12, p. 1983-1991, 2016.

HERMUCHE, P.M.; SANO, E.E. Identificação da Floresta Estacional Decidual no Vão do Paranã, Estado de Goiás, a partir da análise da reflectância acumulada de imagens do sensor ETM+/ Landsat-7. Revista Brasileira de Cartografia, v.63, p.415-425, 2011..

INSTITUTO BRASILEIRO DE GEOGRAFIA E ESTATÍSTICA (IBGE). Manual Técnico de Uso da Terra. $3^{\mathrm{a}}$ ed. Brasília.

INSTITUTO NACIONAL DE COLONIZAÇÃO E REFORMA AGRÁRIA (INCRA). Informações Gerais sobre os assentamentos da Reforma Agrária. 2017. Disponível em: <painel.incra.gov.br/sistemas/index.php>. Acesso em 08.mai.2017.

MCLAUGHLIN, J., MCKENNA, J. Property and development: building the essential infrastructure. Land Use Policy, v.15, n. 1, p. 1-2, 1998.

NASCIMENTO, E.R.P.; SANO, E. E. Identificação de cerrado rupestre por meio de imagens multitemporais do Landsat: proposta metodológica. Sociedade e Natureza, v. 22, p.93-106, 2010.

NOVACK, T.; KUX, H.; FEITOSA, R.Q.; COSTA, G.A.O.P. A knowledge-based, transferable approach for block-based urban land-use classification. International Journal of Remote Sensing, v.35, p.4739-4757, 2014. DOI: 10.1080/01431161.2014.921943.

OLIVEIRA, L. T. G. Land regularization in Brazil and the global land grab. Development and Change, v. 44, n. 2, p. 261-283, 2013.

PACHECO, P. Agrarian reform in the Brazilian Amazon: its implications for land distribution and deforestation. World development, v. 37, n. 8, p. 1337-1347, 2009.

PASSO, D.P.; BIAS, E. de S.; BRITES, R.S.; COSTA, G.A.O.P. Uso do sistema InterIMAGE para a identificação de alvos urbanos em imagens do satélite Worldview II. Revista Brasileira de Cartografia, v.65, n.6, p.1211-1221, 2013.

QGIS. Comunidade QGIS Brasil. Disponível em: <qgisbrasil.org>. Acesso em: 05.dez.2016.

SANO, E.E.; ROSA, R.; BRITO, J.L.S.; FERREIRA, L.G. Land cover mapping of the tropical savanna region in Brazil. Environmental Monitoring and Assessment, v. 166, p.113-1242010. DOI: 10.1007/s10661- 
009-0988-4.

SCHOWENGERDT, R. A. Techniques for image processing and classifications in remote sensing. Academic Press, 2012.

\section{Biografia do autor principal}

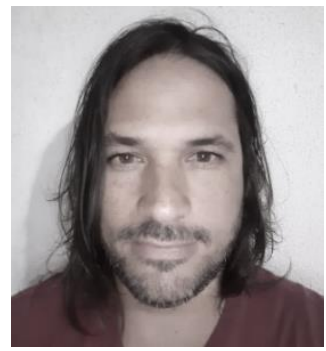

Ramon Chaves de Araújo, Brasília/DF - 1982. Engenheiro Agrônomo (Universidade Federal de Viçosa-MG), Especialista em Geoprocessamento Ambiental (Instituto de Geociências/IG - Universidade de Brasília). Servidor Público Federal do Instituto Nacional de Colonização e Reforma Agrária desde 2006, lotado na Coordenaçao Geral de Cartografia / Divisão de Geomensura com atuação na gestão da política de georreferenciamento / certificação de imóveis rurais. 